\title{
Superpixel Estimation for Hyperspectral Imagery
}

\author{
Pegah Massoudifar, Anand Rangarajan, Paul Gader \\ Department of CISE, University of Florida, Gainesville, FL 32611, USA \\ $\{$ pegah, anand, pgader\}@cise.ufl.edu
}

\begin{abstract}
In the past decade, there has been a growing need for machine learning and computer vision components (segmentation, classification) in the hyperspectral imaging domain. Due to the complexity and size of hyperspectral imagery and the enormous number of wavelength channels, the need for combining compact representations with image segmentation and superpixel estimation has emerged in this area. Here, we present an approach to superpixel estimation in hyperspectral images by adapting the well known UCM approach to hyperspectral volumes. This approach benefits from the channel information at each pixel of the hyperspectral image while obtaining a compact representation of the hyperspectral volume using principal component analysis. Our experimental evaluation demonstrates that the additional information of spectral channels will substantially improve superpixel estimation from a single "monochromatic" channel. Furthermore, superpixel estimation performed on the compact hyperspectral representation outperforms the same when executed on the entire volume.
\end{abstract}

\section{Introduction}

With the ramping up of hyperspectral imaging technologies in the past twenty years, there is now widespread interest in the application of computer vision and machine learning techniques in this domain. Regardless of whether data are specifically acquired in the infra-red, microwave or the ultra-violet ends of the spectrum, the foci of information processing of hyperspectral images largely remain the same: endmember extraction, abundance estimation and segmentation are typical examples. Given these overarching goals, it is somewhat surprising to see the lack of application of standard computer vision modules in the hyperspectral domain, with superpixel segmentation being an obvious example.

In the past decade, we have witnessed the sparing use of segmentation approaches such as graph cuts [13] and belief propagation [10] in the hyperspectral domain. The need for these is seemingly easy to motivate. Hyperspectral imagery of complex scenes benefit from region specific estimation of endmembers (followed by abundance estimation) and this calls for hyperspectral image segmentation prior to (and concomitant with) endmember extraction. Unfortunately, given the remote sensing aspect of hyperspectral acquisition, the paucity of object models makes it difficult to perform model based segmentation. Completely unsupervised segmentation is even more challenging with the lack of ground truth compounding the difficulty. Given these challenges, it is quite surprising to see the lack of application of superpixel estimation in the hyperspectral domain. To be clear, we use the term superpixel estimation to refer to the process of super-pixellization-a process by which nonuniform image tessellations are obtained-which should not be confused with the much more difficult task of image segmentation. We believe that hyperspectral superpixels are a useful intermediate representation and can significantly improve downstream modules such as endmember extraction. We further believe that hyperspectral superpixels are more efficient to compute than segmentation per se while being helpful for abundance estimation etc.

Insofar as we have identified a clear cut need-the estimation of hyperspectral superpixels, the rest of this paper is entirely straightforward. We first review the state of the art of hyperspectral superpixel estimation finding a lack of a lot of previous work in this area. This considerably simplifies our task-we essentially survey the set of superpixel estimation methods for color images and set about adapting the best of breed to the hyperspectral domain. The result is HYP-UCM - an application of the popular ultrametric contour map (UCM) approach for hyperspectral imagery.

\section{Literature Review}

Hyperspectral imagery was introduced at National Aeronautics and Space Administration (NASA)'s Jet Propulsion Laboratory and since then NASA has gathered enormous amount of images with hundreds of spectral channels containing either visible or near infrared spectra of the reflectance of light [6]. Therefore each pixel of the hyperspectral image can be considered a spectral function which 
characterizes the underlying objects in the image.

There has been a growing need for image segmentation in the hyperspectral area over the past decade with recent work borrowing from machine learning and computer vision approaches. Some methods have exploited the correlation between spatial and spectral neighbors and used support vector machine (SVM) training [4], minimum spanning forests [15], classification using the watershed algorithm [16] or multinomial logistic regression with active learning [8]. The work in [10] addressed the problem of hyperspectral classification by using loopy belief propagation and most recently, composite kernels have been generalized to be used for classification in [11]. Graph-cuts based segmentation for hyperspectral images (GRENDEL) [13] has been used as an important step for a piece-wise convex unmixing of hyperspectral images.

Needless to say, there has been an enormous amount of work on superpixel estimation in computer vision. Newer approaches have used learning techniques to combine brightness, texture and color information from the input image [3]. Some approaches have combined information at multiple scales [12] and a variety of other novel approaches have been proposed [14, 2, 5]. The approach most suited to our present work is the ultrametric contour map (UCM) which uses brightness, color and texture cues to estimate the probability of existence of a boundary at each pixel in the image [1] —essentially a multiscale extension of the work in [12].

Very little previous work exists for the problem of superpixel estimation for hyperspectral images. In [17], superpixels have been computed with a graph based approach on a grid using the sum of squared differences between neighboring pixels and later in [18], superpixels have been used for endmember detection and unmixing. To summarize, we have not yet seen the application of the latest state of the art superpixel estimation approaches in the hyperspectral domain-hence the motivation for the present work.

\section{Methodology}

Given the development thus far, the methodology for superpixel estimation is entirely straightforward. We first provide an intuitive overview of our approach to superpixel estimation followed by mathematical development.

It is common to refer to the channel information available at each hyperspectral pixel as a feature vector with intensity information available at each wavelength. This is entirely misleading since (i) the wavelengths are usually close to each other and (ii) the set of wavelengths possess linear ordering. For these reasons, it may actually be beneficial to consider the hyperspectral image as a 3D volume (by performing interpolation along space and wavelength dimensions). We have not chosen this route in this paper (setting this larger goal aside for future work). However, the fact that nearby wavelengths carry similar reflectance information is obviously known to researchers in the hyperspectral domain but to the best of our knowledge has not been utilized in superpixel estimation. Based on this observation, we conjecture that feature extraction (oriented filter banks etc.) at every wavelength is highly redundant calling for the construction of more compact representations of the $3 \mathrm{D}$ reflectance distribution prior to feature extraction.

In this work, we have elected to use principal component analysis (PCA) to obtain a compact representation of the hyperspectral volume. While more recently, sparse and overcomplete representations have become available, there is no previous work on superpixel estimation from PCA driven compact representations. Our first step therefore is to perform PCA on the hyperspectral volume followed by feature extraction and superpixel estimation. These are next described.

The steps in superpixel estimation are as follows: (i) compact representation using PCA, (ii) feature extraction using oriented scale space derivatives, (iii) graph construction, (iv) eigenvector computation and (v) oriented watershed transform to produce non-uniform tessellations. While this sequence is somewhat of a simplification, the major steps have been highlighted. The sequence follows the well known UCM approach for the most part but obviously tailored to hyperspectral imagery. The UCM approach combines local and global contour information by combining information from the original image and weighted graph eigenvector "images." In the hyperspectral case, in addition to the regular channels, we also have PCA wavelength channels which provide more contour information. We now detail the individual steps in the overall sequence:

Step 1: Scale space feature extraction from brightness, texture and wavelength channels: We execute oriented Gaussian derivative filters at multiple scales to obtain

$$
I_{\text {local }}(\mathbf{x}, \theta)=\sum_{\lambda} \sum_{s(\lambda)} \sum_{i(\lambda)} w_{i(\lambda), s(\lambda)} G(\mathbf{x}, \theta ; \sigma(i(\lambda), s(\lambda)))
$$

where $\left\{w_{i(\lambda), s(\lambda)}\right\}$ is a set of weights that depend on the channels and scales. The dependence between the Gaussian filters, the scales and the wavelengths can range from the simple to the complex. Here, we have just executed the filters at multiple scales, brightness, textures and wavelengths. This results in a set of local features at different orientations which integrates information from all wavelengths. Note that while (1) is agnostic regarding the chosen wavelengths, in our case these correspond to the hyperspectral principal components as detailed in the next section. The inner wights in each channels have been learned using a set of channels of Pavia dataset wavelengths as training images and the outer weights of each PCA channel is the square root of the eigenvector related to that specific channel. 
Step 2: Weighted graph construction: A weighted graph (for the purposes of eigenvector computation) is constructed using the local filter responses above. Following the UCM strategy, pixels within a certain distance of each other are linked by a weighted edge using the relation

$$
W(\mathbf{x}, \mathbf{y})=\exp \left\{-\alpha \max _{\mathbf{z}(\mathbf{x}, \mathbf{y})} \max _{\theta} I_{\text {local }}(\mathbf{z}(\mathbf{x}, \mathbf{y}), \theta)\right\}
$$

where $\alpha$ is a constant and $\mathbf{z}(\mathbf{x}, \mathbf{y})$ is any point lying on the line segment connecting $\mathbf{x}$ and $\mathbf{y}$.

Step 3: Eigenvector computation from the weighted graph $W(\mathbf{x}, \mathbf{y})$ : Following the standard strategy of spectral clustering, we compute the top eigenvectors of the weighted graph. Since these eigenvectors are in location space, the result is a set $\left\{e_{k}(\mathbf{x})\right\}$ (usually rescaled using the eigenvalues of the weighted graph).

Step 4: Spectral information obtained from the top $K$ eigenvectors: Since gradient information computed from the scaled eigenvectors can be expected to contain complementary spectral information [1], we execute a set of gradient operations in different orientations to get

$$
I_{\text {spectral }}(\mathbf{x}, \theta)=\sum_{k} \nabla_{\theta}\left(e_{k}(\mathbf{x})\right)
$$

A point to note here is that we considered separately computing eigenvectors for each hyperspectral PCA channel and then combining all this global information into a single descriptor at each pixel. While this needs more investigation, at this early stage of hyperspectral superpixel estimation, we decided in the interest of simplicity to use the integrated eigenvectors instead. We plan to revisit this issue in the future.

Step 5: Combination of local and spectral information: We linearly combine the information in (11) and in (3) to obtain the final, global contour probability measure (Fig. 2) A free parameter is used for the combination and this needs more careful validation (which we reserve for future work).

Step 6: Oriented watershed transform applied to the global contour measure: Since the global contour probability map may not always be closed and therefore may not divide the image into regions, we require another operation to extract closed contours. We have used the Oriented Watershed Transform (OWT) [1] to construct closed contours. Here, the orientation that maximizes the response of the contour detection approach is used to construct a set of regions and further build a hierarchical region tree from the input contours. Real valued weights are associated with each possible segmentation based on their likelihood to be a true boundary. For a specific threshold, the set of the resulting closed contours can be seen either as a segmentation or as the output of the super-pixellization. Further it can be

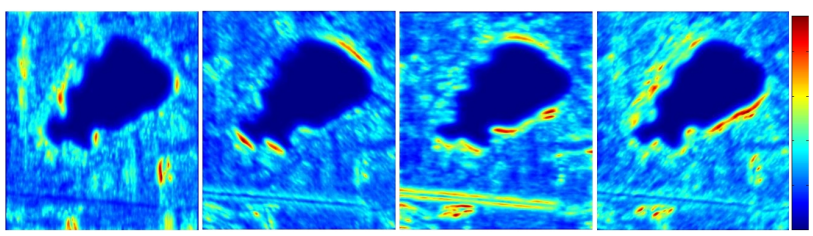

(a) NEON data
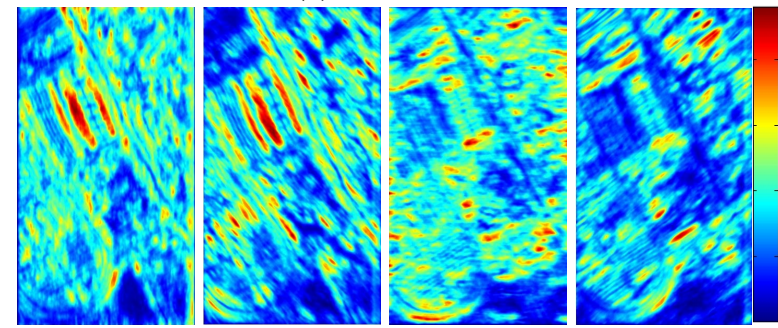

(b) Pavia data

Figure 2. $I_{\text {global }}(\mathbf{x}, \theta)$ : from left to right for four different orientations: $\theta=\frac{\pi}{2}, \frac{\pi}{4}, 0, \frac{3 \pi}{4}$.

seen that the uncertainty of a segmentation can be represented. At low thresholds, the image can be oversegmented respecting even very least probable boundaries and as you make the threshold higher only very strong boundaries survive (Fig. 4). This has the benefit of introducing a trade off between the extreme ends of the segmentation.

\section{Experimental Results}

In the following, HYP-UCM is tested using two real hyperspectral datasets, NEON and Pavia which are explained in the following:

A. NEON: The National Ecological Observatory Network (NEON) has conducted a series of airborne flights and supporting ground measurements in two study areas located near Gainesville, Florida since 2010. Major plant communities exist within the region and these diverse targets are populated by sandhill, mixed forests, basin swamp, basin marsh, marsh lake, etc. [9]. We conducted manual preprocessing to remove the very noisy bands and also rescaled the whole image between $[0,1]$. The image used in the experiments has $358 \times 317$ pixels with 171 different bands.

B. Pavia: This data set was collected over an urban area of Pavia, in northern Italy by the ROSIS spectrometer on July 8,2002 [19]. The image contains $610 \times 341$ pixels with 103 bands. The ROSIS sensor collected data over the $430-850 \mathrm{~nm}$ wavelength range at a $4 \mathrm{~nm}$ spectral sampling interval. The image has been atmospherically corrected by the German Remote Sensing Data Center of the German Aerospace Center (DLR) [7]. The image contains both natural and urban regions.

We have used PCA to select the most informative components of the data to give the PCA channels as the input to HYP-UCM. Fig. 3 shows that that each of the 3 different components emphasizes specific details different from 

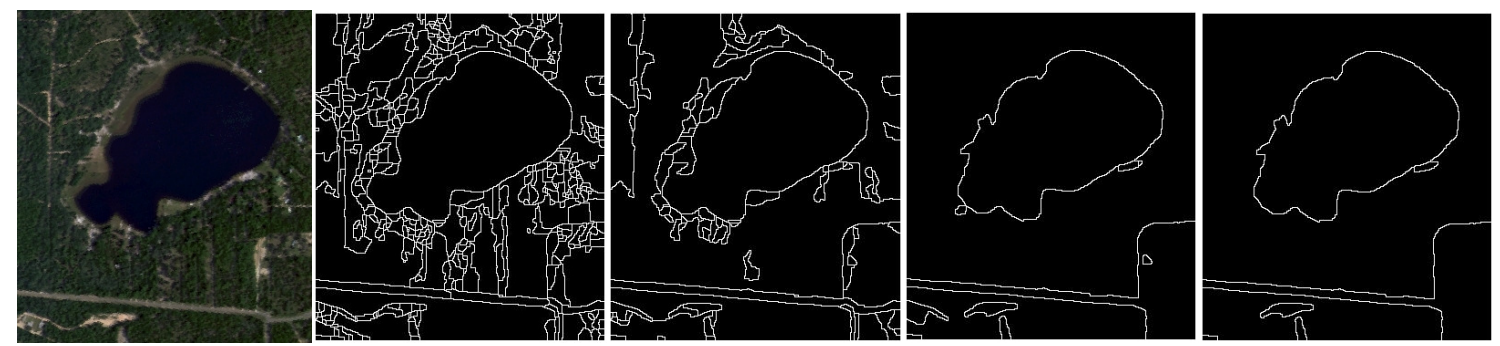

(a) NEON image
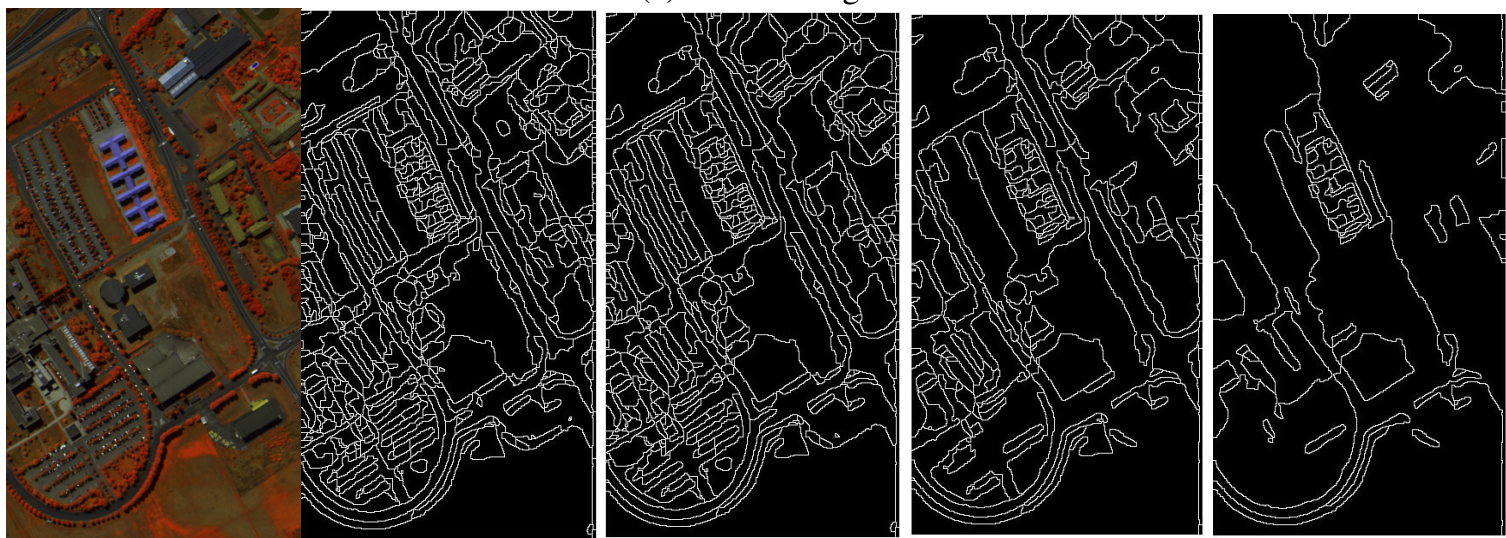

(b) Pavia image

Figure 1. Hierarchical Results for NEON (a) and Pavia (b): From left to right the threshold becomes higher and the weaker boundaries will be omitted. Far left image is oversegmented and as we go to the right only the strong boundaries survive. As the threshold rises the results still remain reasonable. For the NEON image, the main lake at the top and the road at the bottom have been successfully detected whereas in Fig. 5 a) the road has been missed. Also for Pavia (b), the strong boundaries of the buildings have all survived as the threshold gets higher compared to Fig. 5 b).

the other-a key reason why PCA is useful in this context. Future work will focus on dictionary learning to determine better sparse representations. We have used the wavelength channels related to the highest eigenvalues and for each specific PCA component, 6 different inner channels are used. All the weights of the 6 channels are learned using training data and the outer weight assigned to each PCA component is the square root of the corresponding eigenvalue.

Fig. 4 contains hierarchical results of HYP-UCM for NEON and Pavia. At very small thresholds, the results get oversegmented and as we go to the higher thresholds only the strong boundaries survive. It can be seen in Fig. 4 that when the threshold gets larger, the result still remains reasonable in comparison to the case without using PCA (Fig. 5). For NEON, the main lake at the top and the road at the bottom have been successfully detected whereas in Fig. 5(a) the road has been missed. Also for Pavia (b), the strong boundaries of the buildings have all survived as the threshold gets higher compared to Fig. 5(b) where at the higher thresholds the results look completely unreasonable.

Moreover, our results show that using UCM with the RGB version of each of the hyperspectral images as input
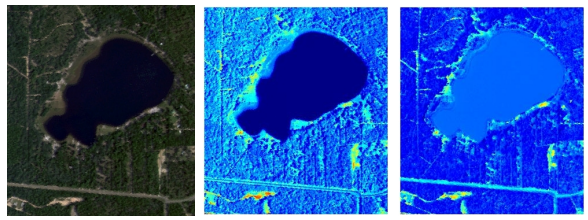

(a) NEON data
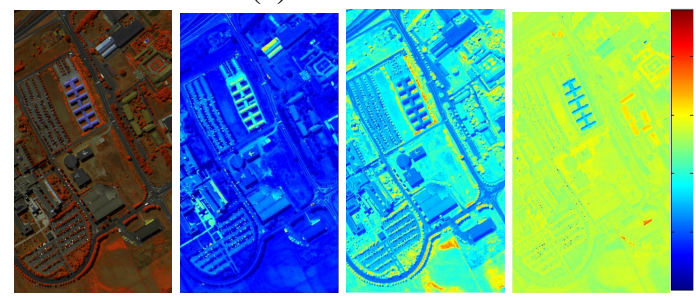

(b) Pavia data

Figure 3. The results of PCA for (a) NEON and (b) Pavia. From left to right: The input image and the first, second and third principal components of the NEON image. It can be seen that each of the components emphasizes specific details different from the others which is the key reason why we have used PCA to choose a certain number of hyperspectral bands.

leads to less accurate results compared to the case of HYPUCM with PCA (Fig. 6] and Fig.77. 

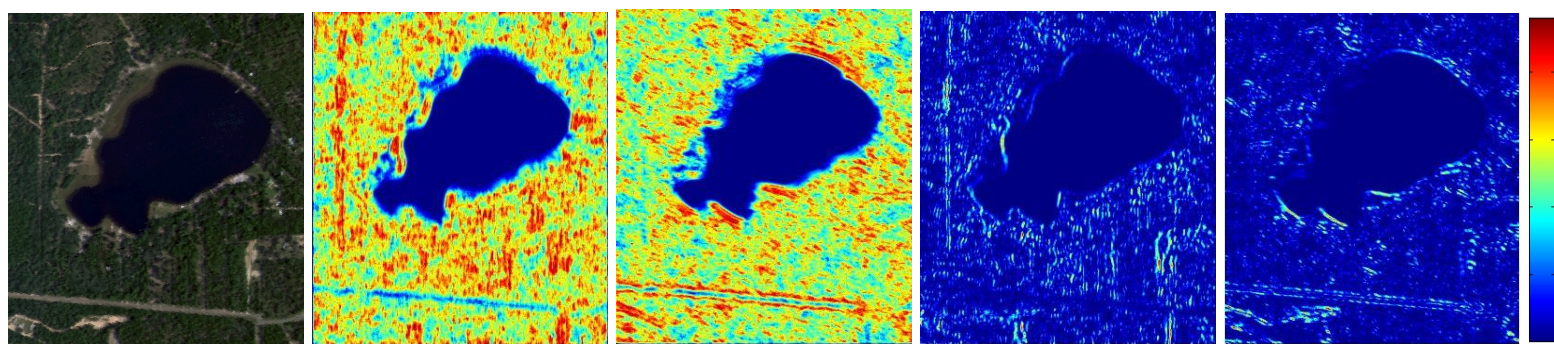

(a) NEON data
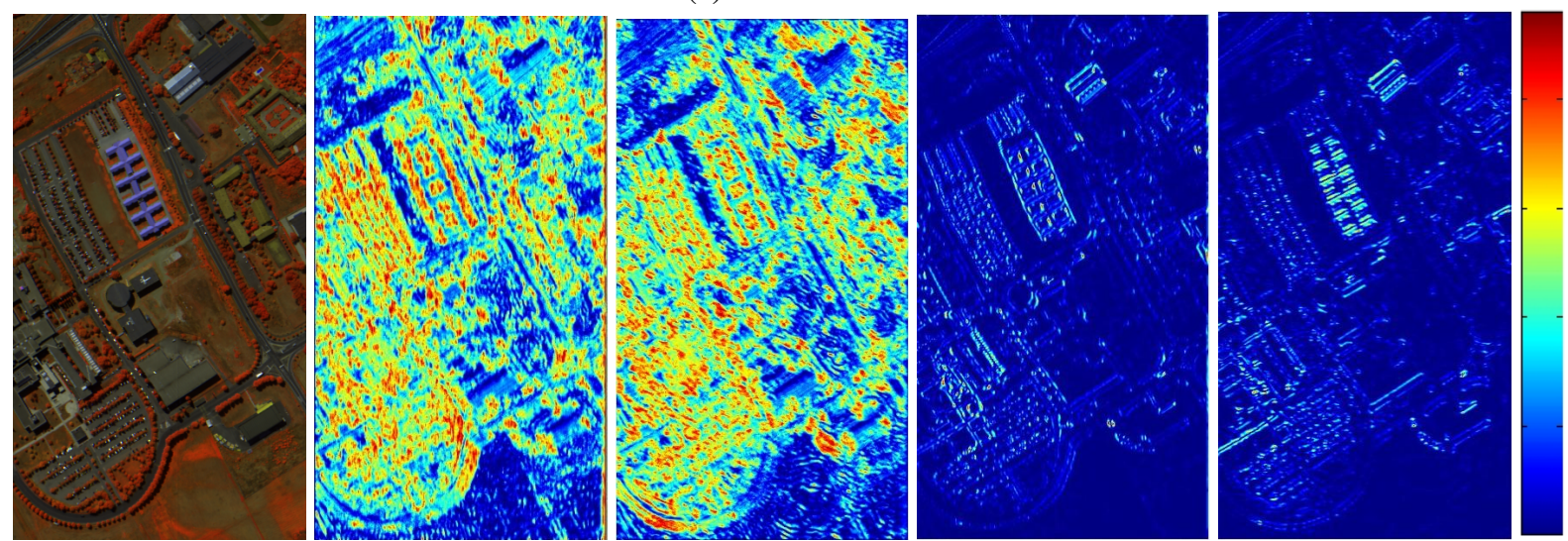

(b) Pavia data

Figure 4. 4 chosen Channels for one wavelength of NEON (a) and Pavia (b): From left to right the first image is the input and the 2 middle left images are two chosen brightness channels of the first PCA channel for $\theta=\frac{\pi}{2}$ and $\theta=\frac{\pi}{8}$ and the two right most images are two chosen texture channels for $\theta=\frac{\pi}{2}$ and $\theta=\frac{\pi}{8}$.
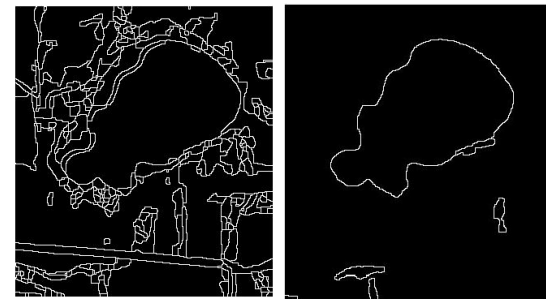

(a) NEON data
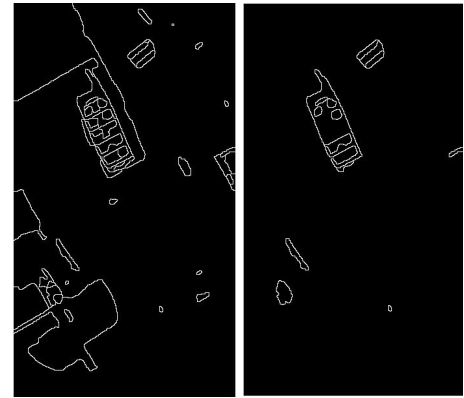

(b) Pavia data

Figure 5. As the threshold is increased, the results of using all of the bands do not produce reasonable contours. The road in the bottom and some other boundaries around the lake have been completely missed. In (b) the boundaries that survived are not the strongest.

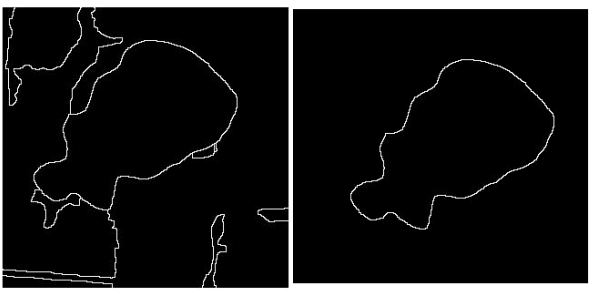

(a) UCM using the RGB version of the NEON data

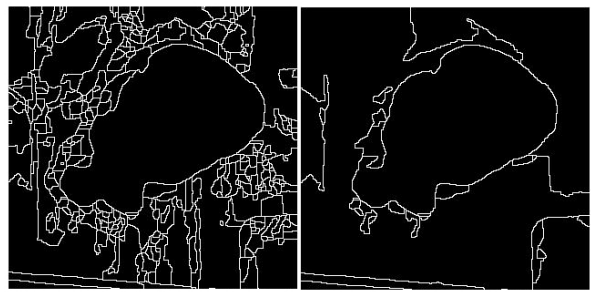

(b) HYP-UCM using the the PCA channels of NEON

Figure 6. Comparison of superpixel estimation results from the input RGB image (top row), and the first 10 PCA bands of the hyperspectral image (bottom row), for the extremely low (left column) and extremely high (right column) thresholds. The results in (a) do not contain the main boundaries as the ones in (b). Even at extremely high thresholds, like the left image in (a), the most noticeable detection of UCM is just the very large lake in the center. 


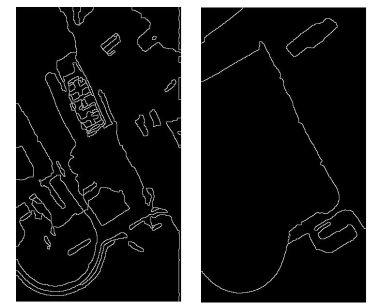

Figure 7. Comparison of superpixel estimation results from the input RGB Pavia image (right), and the first 10 PCA bands of the hyperspectral image (left) for a very high threshold. As the threshold is increased, the RGB result is not as accurate as the hyperspectral.

\section{Conclusion}

In this work, we have presented an extremely straightforward approach to the estimation of superpixels for hyperspectral volumes. After summarizing the paucity of previous work in superpixel estimation in this domain, we justified the decision to adapt and extend the well known UCM approach to hyperspectral imagery. Many issues especially pertinent to hyperspectral images were set aside for future work. For example, the imposition of continuity along the wavelength dimension is a strong constraint and expected to have a big impact on the construction of compact volumetric hyperspectral image representations and is a key direction for future work. Our results clearly indicate that superpixel estimation on compact hyperspectral representations is more efficient than performing the same on the entire volume. At the same time, the additional channel information does improve the superpixels relative to using a single "monochromatic" channel. Since we are well versed in endmember extraction and abundance estimation, we are positioned to adapt standard approaches such as PCOMMEND to non-uniform hyperspectral tessellations thereby leveraging superpixel homogeneity. Finally, we think the time is ripe for more computer vision and machine learning applications and extensions in the hyperspectral domain and look forward to fostering such cross-fertilization

\section{References}

[1] P. Arbelaez, M. Maire, C. Fowlkes, and J. Malik. Contour detection and hierarchical image segmentation. IEEE Trans. Patt. Anal. Mach. Intell., 33(5):898-916, 2011. 2, 3, 3

[2] B. Catanzaro, B.-Y. Su, N. Sundaram, Y. Lee, M. Murphy, and K. Keutzer. Efficient high-quality image contour detection. In IEEE Intl. Conf. Comp. Vision, pages 2381-2388, 2009. 2

[3] P. Dollar, Z. Tu, and S. Belongie. Supervised learning of edges and object boundaries. In IEEE Conf. Comp. Vision and Patt. Recog., volume 2, pages 1964-1971, 2006. 2

[4] M. Fauvel, J. Benediktsson, J. Chanussot, and J. Sveinsson. Spectral and spatial classification of hyperspectral data using SVMs and morphological profiles. IEEE Trans. Geoscience Remote Sensing, 46(11):3804-3814, 2008. 2
[5] B. Fulkerson, A. Vedaldi, and S. Soatto. Class segmentation and object localization with superpixel neighborhoods. In IEEE Intl. Conf. Comp. Vision, pages 670-677, 2009. 2

[6] A. Goetz, G. Vane, J. Solomon, and B. Rock. Imaging spectrometry for earth remote sensing. Science, 228(4704):11471153, 1985. 2

[7] S. Holzwarth, A. Muller, M. Habermeyer, R. Richter, A. Hausold, S. Thiesmann, and P. Strobl. HySensDAIS/ROSIS Imaging Spectrometers at DLR. In Proceedings of the 3rd EARSeL Workshop on Imaging Spectroscopy, pages 3-14, Herrsching, Germany, 2003. 4

[8] M.-D. Iordache, J. Bioucas-Dias, and A. Plaza. Sparse unmixing of hyperspectral data. IEEE Trans. Geoscience Remote Sensing, 49(6):2014-2039, 2011. 2

[9] T. Kampea, K. Krausea, C. Meiera, D. Barnetta, and J. McCorkel. The NEON 2010 airborne pathfinder campaign in Florida. NEON Technical Memo 002, pages 5-11, 2010. 4

[10] J. Li, J. Bioucas-Dias, and A. Plaza. Spectral-spatial classification of hyperspectral data using loopy belief propagation and active learning. IEEE Trans. Geoscience Remote Sensing, 51(2):844-856, 2013. 1,2

[11] J. Li, P. Marpu, A. Plaza, J. Bioucas-Dias, and J. Benediktsson. Generalized composite kernel framework for hyperspectral image classification. IEEE Trans. Geoscience Remote Sensing, pages 1-14, 2013. 2

[12] D. Martin, C. Fowlkes, and J. Malik. Learning to detect natural image boundaries using local brightness color and texture cues. IEEE Trans. Patt. Anal. Mach. Intell., 26(5):530-549, 2004. 2

[13] P. Massoudifar, A. Rangarajan, A. Zare, and P. Gader. An integrated graph cuts segmentation and piece-wise convex unmixing approach for hyperspectral imaging. In IEEE GRSS Workshop Hyperspectral Image Signal Processing: Evolution Remote Sensing, 2014. (accepted). 1, 2

[14] X. Ren, C. Fowlkes, and J. Malik. Scale-invariant contour completion using conditional random fields. In IEEE Intl. Conf. Comp. Vision, volume 2, pages 1214-1221, 2005. 2

[15] Y. Tarabalka, J. Benediktsson, and J. Chanussot. Spectralspatial classification of hyperspectral imagery based on partitional clustering techniques. IEEE Trans. Geoscience Remote Sensing, 47(8):2973-2987, 2009. 2

[16] Y. Tarabalka, J. Chanussot, J. Benediktsson, J. Angulo, and M. Fauvel. Segmentation and classification of hyperspectral data using watershed. In IEEE Intl. Geoscience and Remote Sensing Symposium, volume 3, pages 652-655, 2008. 2

[17] D. Thompson, R. Castano, and M. Gilmore. Sparse superpixel unmixing for exploratory analysis of CRISM hyperspectral images. In IEEE GRSS Workshop Hyperspectral Image and Signal Processing: Evolution in Remote Sensing, pages $1-4,2009$. 2

[18] D. Thompson, L. Mandrake, M. Gilmore, and R. Castaño. Superpixel endmember detection. IEEE Trans. Geoscience Remote Sensing, 48(11):4023-4033, 2010. 2

[19] A. Zare, P. Gader, O. Bchir, and H. Frigui. Piecewise convex multiple-model endmember detection and spectral unmixing. IEEE Trans. Geoscience Remote Sensing, 51(51):2853-2862, 2013. 4 